\title{
5 Kultur, Religion und Suizid
}

\author{
Kristina M. Ritter
}

Suizid ist ein in allen menschlichen Gesellschaften auftretendes Phänomen und zählt weltweit zu den drei häufigsten Todesursachen. Am stärksten betroffen sind in den meisten Kulturen primär ältere Menschen sowie Personen zwischen 15 und 34 Jahren. Zur Erklärung dieses Verhaltens, das auf dem ersten Blick ganz offensichtlich allen evolutionsbiologischen und -psychologischen Regeln zuwiderläuft, wurden zahlreiche biologische, psychologische, psychiatrische und soziologische Theorien aufgestellt. Am häufigsten wird ein Zusammenhang zwischen suizidalem Verhalten und Veränderungen der serotonergen Neurotransmission herausgestrichen (van Heeringen 2003; Courtet et al. 2005; Audenaert et al. 2006; Rujescu u. Giegling 2010). Dabei weisen die meisten Untersuchungen auf einen Zusammenhang zwischen einem präsynaptischen serotonergen Defizit im präfrontalen Cortex (PFC) und einer Disposition für Impulsivität und Aggressivität hin (Mann 1998; Brunner u. Bronisch 1999). Es finden sich Hinweise auf eine Dysregulation des Hypothalamus-HypophysenNebennierenrinden-Systems (HPA-System) bei Personen nach Suizidversuch und Suizidenten hin. Dabei ist einer der konsistentesten Befunde die Non-Suppression von Cortisol im DexamethasonSuppressionstest (Lester 1995). López et al. (1992) fanden eine Erhöhung von Pro-0pio-MelanocortinmRNA (POMC-mRNA) im Hypophysenvorderlappen von Suizidanten. POMC ist ein biosynthetischer Vorläufer von Adreno-corticotropem Hormon (ACTH) und ß-Endorphin. Die Aktivierung von Rezeptoren für Corticotropin-Releasing-Hormon (CRH) induziert die Transkription des POMC-Gens. Diese Befunde sind konsistent mit der Hypothese einer chronischen Hyperaktivität des HPA-Systems bei suizidalen Patienten.

Es mehren sich Hinweise, dass genetische Risikofaktoren für suizidales Verhalten existieren (Bondy et al. 2006; Mann et al. 2009; Tsai et al. 2010). Dies ergibt sich sowohl aus Familien- und Zwillingsuntersuchungen (Voracek $u$. 
Loibl 2007; Brent et al. 2008; Pedersen u. Fiske 2010), als auch aus molekulargenetischen Studien (Souery et al. 2003; Arango et al. 2003) und aus dem Vergleich der Suizidraten von Migrantenpopulationen mit den Bevölkerungen des Auswanderungs- und des Aufnahmelandes (Voracek u. Loibl 2008).

Von den psychologischen bzw. psychoanalytischen Erklärungsmodellen sind die Aggressionstheorie von Freud und Menninger, sowie die Arbeiten von Ringel zur suizidalen Entwicklung des Individuums am bekanntesten geworden. $\mathrm{Zu}$ den neueren psychoanalytischen Erklärungsmodellen gehören die Arbeiten von Henseler (2000), der Suizid als das Resultat narzisstischer Krisen beschreibt. Allen diesen Modellen ist gemeinsam, dass sie sich auf Gesellschaften der westlich-abendländischen Kultur beziehen weshalb die Reichweite der Aussagen begrenzt ist.

Ende des 19. Jahrhunderts begannen sich die Sozialwissenschaften mit den gesellschaftlichen Ursachen des Suizids auseinanderzusetzen Einer Vorreiter war der bedeutenden Soziologe, Ethnologe und Religionswissenschaftler Emil Durkheim. Basierend auf seiner bekannten Suizidtypologie (egoistischer, altruistischer, anomischer und fatalistischer Selbstmord) entstanden in den darauffolgenden Jahrzehnten weitere bedeutsame soziologische Erklärungsmodelle. Cibbs und Martin (1964) sahen einen Zusammenhang zwischen niedriger Statusintegration und erhöhter Suizidneigung. Maurice Halbwachs (1930) interpretierte die ansteigenden Suizidraten als Folgeerscheinung der Veränderung der traditionellen Lebensweisen in einer zunehmend komplexeren, urbanen Industriegesellschaft. 2001 stellte der Soziologe Feldmann eine weitere Typologie des Suizids auf (normativer, instrumenteller, rationaler und expressiver Suizid), die sich auf Durkheim stützt.

Neben biologischen, psychologischen und soziologischen Faktoren spielen psychische Erkrankungen eine ganz entscheidende Rolle. Eine Metaanalyse von 27 psychologischen Autopsiestudien ergab dabei folgende Rangordnung (s. Tab. 8).

\section{Tab. 8 Suizid und psychische Erkrankungen (\%). Metaanalyse von psychologischen} Autopsiestudien, verglichen mit Kontrollgruppen (nach Arsenault-Lapierre et al. 2004)

\begin{tabular}{|lcc}
\hline diagnostische Kategorie & Odds Ratio & $95 \% \mathrm{Cl}$ \\
\hline depressive Erkrankungen & 6,2 & $5,4-8,1$ \\
\hline bipolare Erkrankungen & 3,0 & $1,5-9,9$ \\
\hline Substanzmissbrauch & 3,5 & $3,1-4,5$ \\
\hline Psychosen & 6,6 & $3,9-11,9$ \\
\hline Persönlichkeitsstörungen & 4,5 & $3,5-6,4$ \\
\hline Angststörungen & 2,4 & $1,7-3,6$ \\
\hline Anpassungsstörungen & 1,3 & $0,7-2,4$ \\
\hline irgendeine psychische Erkrankung & 10,5 & $9,6-13,4$ \\
\hline
\end{tabular}


Am häufigsten fanden sich im Vorfeld von Suiziden psychotische und affektive Erkrankungen, gefolgt Persönlichkeitsstörungen. Aber auch Patienten mit Substanzmissbrauch und bipolare Erkrankungen haben im Vergleich zur gesunden Bevölkerung ein deutliches höheres Risiko, durch Selbsttötung aus dem Leben zu scheiden. Zahlreiche anders methodisch ausgerichtete Studien bestätigen dieses Ergebnis. Vor allem Depressionen, Angststörungen, psychotische Erkrankungen und Borderline- oder narzisstische Persönlichkeitsstörungen sind häufig von suizidalen Verhaltensweisen begleitet (Cheng 1995, 1997; Arsenault-Lapierre et al. 2004; Hawgood u. De Leo 2008; Krysinska u. Lester 2010; Hor u. Taylor 2010).

Alle bisher besprochenen Erklärungsansätze können allerdings die deutlich voneinander abweichenden nationalen Suizidraten nicht erklären. Der vorliegende Betrag untersucht daher die Zusammenhänge zwischen Suizid, Kultur und Religion. Er umfasst folgende Themenbereiche:

1. Suizidraten in Nationalstaaten und indigenen Gesellschaften

2. Biologische und soziokulturelle Erklärungsmodelle der nationalen Suizidraten

3. Kulturspezifische suizidale Verhaltensweisen

4. Religion und Suizid

5. Soziokultureller Umgang mit Suizid

Darüber hinaus werden eigene Berechnungen von aggregierten Daten über den Einfluss soziodemographischer und konfessioneller Faktoren auf die Höhe der nationalen Suizidraten vorgestellt.

\subsection{Suizidraten in Nationalstaaten und in indigenen Gesellschaften}

Die Daten der World Health Organisation weisen seit langem darauf hin, dass die nationalen Suizidraten weltweit stark voneinander abweichen (s. Abb. 19).

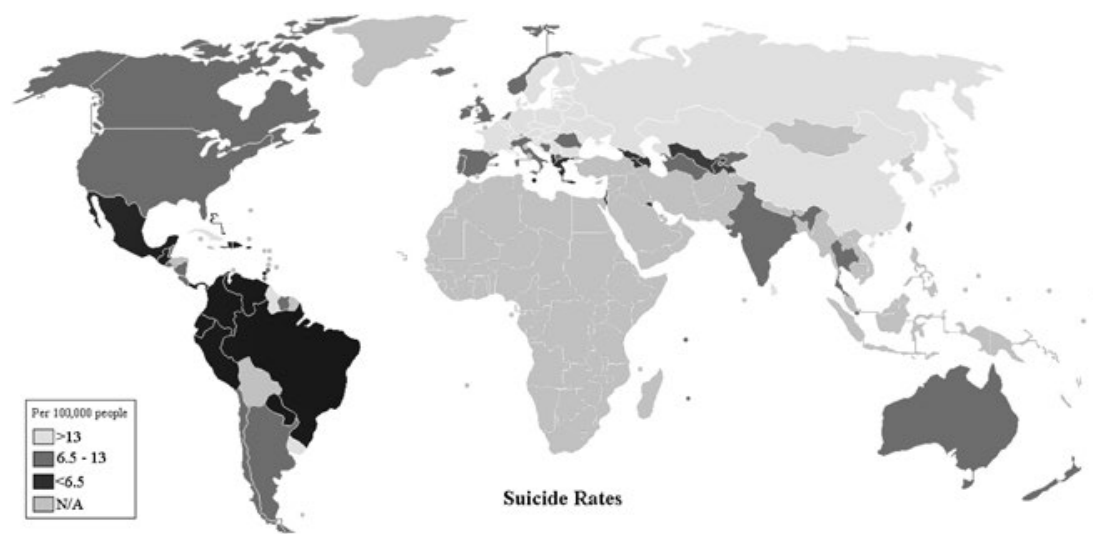

Abb. 19 Nationale Suizidraten 
Tab. 9 Historische Entwicklung nationaler Suizidraten (nach Lester 2008a)

\begin{tabular}{|c|c|c|c|}
\hline Land & 1901 & 1950 & 1990 \\
\hline Australien & 11,9 & 9,3 & 12,9 \\
\hline Belgien & 12,9 & 12,9 & 19,0 \\
\hline England & 9,6 & 10,2 & 7,8 \\
\hline Finnland & 6,1 & 15,6 & 30,3 \\
\hline Deutschland & 20,8 & 18,8 & 17,5 \\
\hline Italien & 6,2 & 6,5 & 7,6 \\
\hline Niederlande & 5,8 & 5,5 & 9,7 \\
\hline Neuseeland & 10,2 & 9,2 & 13,5 \\
\hline Norwegen & 5,5 & 7,4 & 15,5 \\
\hline Portugal & 4,3 & 10,1 & 8,8 \\
\hline Spanien & 2,0 & 5,4 & 7,5 \\
\hline Schweden & 13,1 & 14,9 & 17,2 \\
\hline Schweiz & 22,4 & 23,5 & 21,9 \\
\hline USA & 10,4 & 11,4 & 12,4 \\
\hline
\end{tabular}

So zeigt sich beispielsweise im Jahr 2000 eine Suizidrate von 80,4 pro 100.000 Einwohner bei Männern in Litauen im Vergleich zu o,1 bei Männern in Ägypten bzw. für Frauen in Litauen 16, 9 pro 100.00o Einwohner im Vergleich zu o, o für Frauen in Ägypten. Einschränkend muss allerdings festgestellt werden, dass aufgrund der fehlenden Suizidstatistiken einiger Länder aus Afrika, Asien und Lateinamerika kann die WHO (World Health Organization) nur begrenzt über weltweite Tendenzen von Suizidalität Aussagen treffen. Mit der Ausnahme von China haben Männer eine höhere Suizidrate als Frauen (Ji et al. 2001; Yip et al. 2005; Canetto 2008).

Während die nationalen Suizidraten teilweise erheblich voneinander abweichen, erweisen sich die Selbsttötungsraten in einzelnen Ländern häufig als relativ stabil (s. Tab. 9).

Lester (2008a) fand allerdings alle Varianten: während sich die Suizidraten in Australien, England, Deutschland, Italien, Portugal, Schweiz und den USA zwischen 1901 und 1990 relativ stabil waren, stiegen sie im selben Zeitraum in Belgien, Finnland, Neuseeland, den Niederlanden, Norwegen und Schweden deutlich an. Die nationalen Unterschiede waren jedoch deutlich größer als die Schwankungen über die Zeitachse.

Nur in einigen wenigen Gesellschaften, meist eher in isoliert lebenden Gruppen, war Suizid unbekannt. So etwa bei den Zunis im Süden der USA, der indigenen Bevölkerung der Andamanen und bei einigen australischen Stämmen (Pfeiffer 1994). Neuere Studien (s. Abb. 20) weisen allerdings darauf hin, dass 


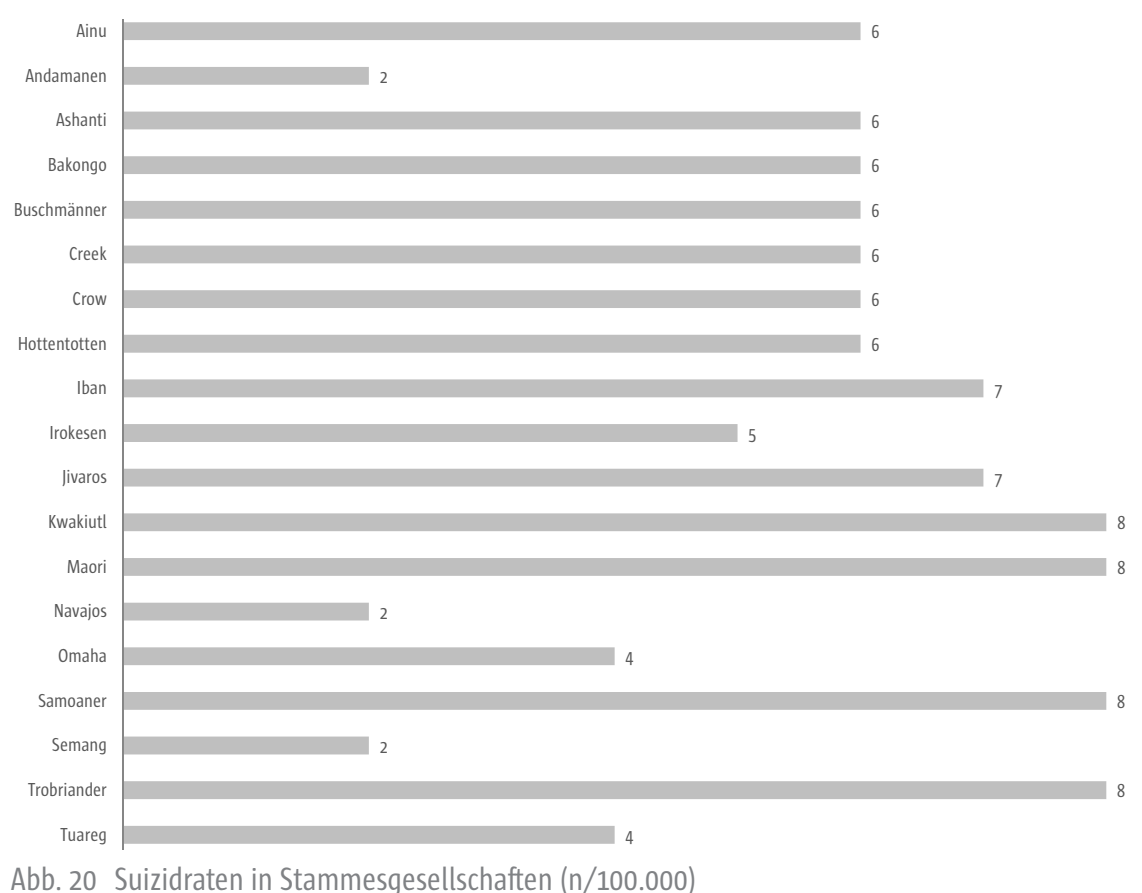

sich auch in verschiedensten Stammesgesellschaften eine Suizidrate von zwischen 2 bis 8 Personen/10o.ooo Einwohner nachweisen lässt (Ogden et al. 1970; Conrad et al. 1974; Lester 2007; Mullany et al. 2009).

Diese Raten finden sich bei durchaus kulturell unterschiedlichen Ethnien aller Kontinente, sowohl bei Jäger und Sammlern als auch in Ackerbaugesellschaften.

Der Median der Suizidraten von 99 Staaten, von denen diese Daten vorliegen, beträgt dazu im Vergleich 9,7/100.ooo Einwohner und liegt damit nur unwesentlich höher als die Suizidrate der indigenen Populationen.

\subsection{Biologische und soziokulturelle Erklärungsmodelle der nationalen Suizidraten}

Nach Lester (2004) existiert ein negativer Zusammenhang zwischen Blutgruppe o und den nationalen Suizidraten. Dieses Ergebnis konnte von uns durch aktuelle Berechnung anhand der Daten aus 41 Ländern bestätigt werden (s. Abb. 21).

Ebenso findet sich ein Zusammenhang zwischen der Häufigkeit von Serotonintransportergen-Polymorphismen und den nationalen Suizidraten von Männern (Spearman-Rho $=, 604^{* *}$ ), bei Frauen findet seich hingegen keine Assoziation zwischen 5-HTTPLR \%L und Suizidraten (s. Abb. 22). 


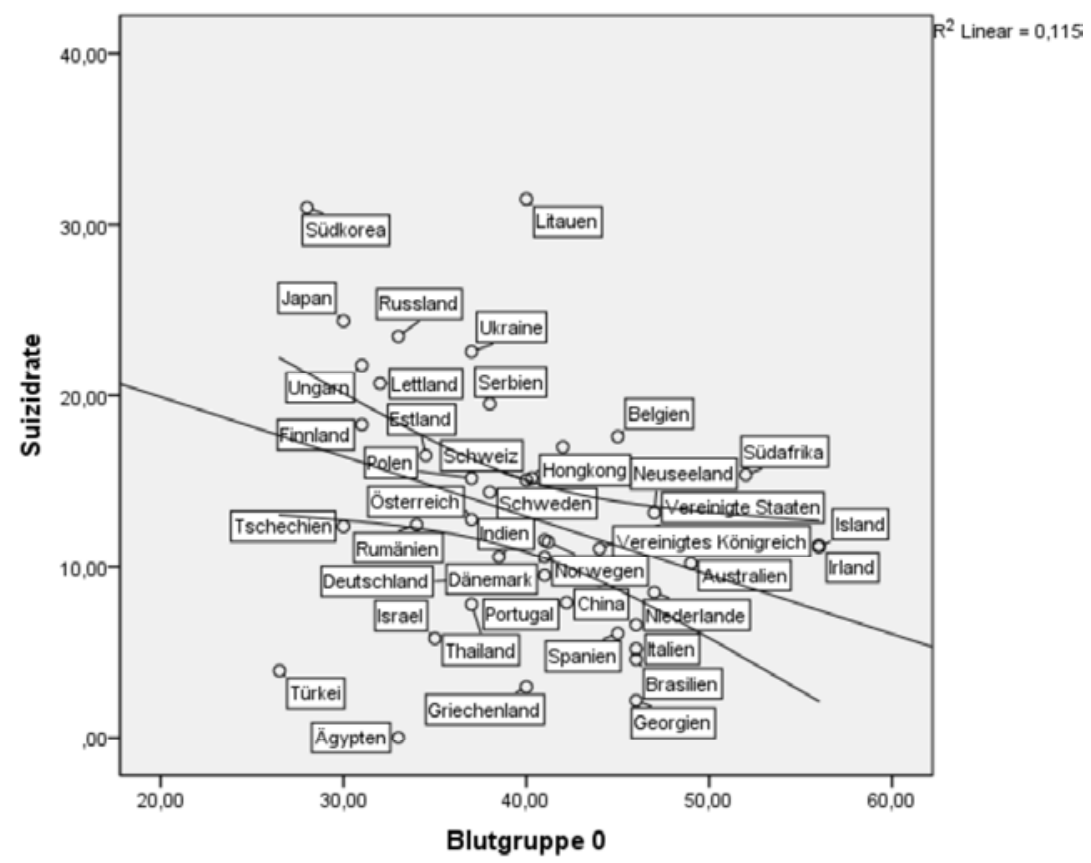

Abb. 21 Nationale Suizidraten und der prozentuelle Anteil an Blutgruppe 0

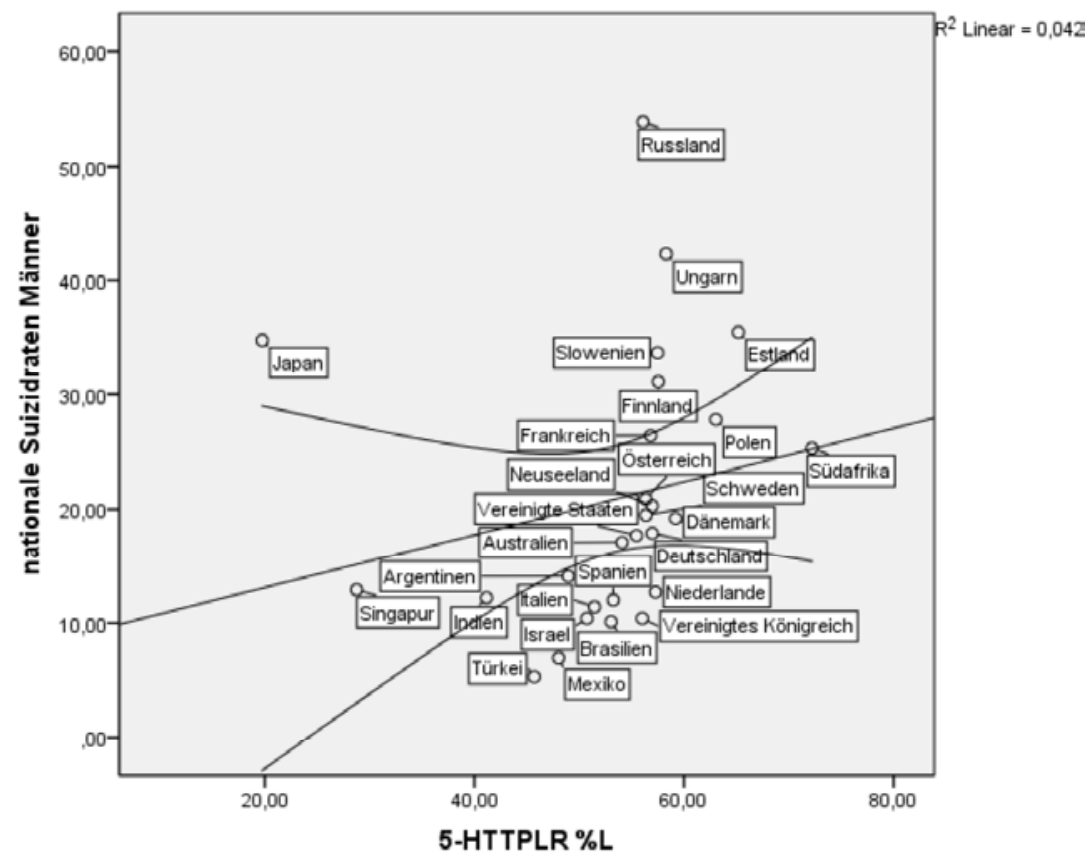

Abb. 22 Nationale Suizidraten und der prozentuelle Anteil an dem SerotonintransportergenPolymorphismus 5-HTTPLR \% L (Daten zur Häufigkeit des SerotonintransportergenPolymorphismus aus Chiao u. Blizinsky 2010) 
Andere Hinweise auf eine populationsgenetische Grundlage sind Untersuchungen an Migrantenpopulationen: Finnisch-ugrische Populationen wie Finnen, Litauer oder Ungarn weisen hohe Suizidraten auf. Migranten aus diesen Ländern zeigten noch nach zwei Generationen ähnlich hohe Suizidraten wie die Bevölkerung in der Heimat (Voracek et al. 2007).

Um die unterschiedlichen nationalen Suizidraten zu erklären, haben Conklin und Simpson (1987) eine viele soziodemographischen und wirtschaftlichen Variablen einschließende Faktorenanalyse durchgeführt. Dabei wurden zwei Cluster mit niedrigen Suizidraten gefunden. Cluster 1 enthielt Länder, in denen die islamische Religion dominant war, Cluster 2 wirtschaftlich unterentwickelte Staaten.

In der Zwischenzeit sind von der WHO und der UNO regelmäßig zahlreiche aggregierte soziodemographische Daten über einen Großteil der Nationaldaten zugänglich gemacht worden. Von 99 Staaten liegen Daten über Suizidraten vor. Der Medianwert liegt bei 9,7 pro 100.ooo Einwohner (25 Perzentil: 4,3, 75 Perzentil: 15,1). Die höchsten Suizidraten weisen die ehemaligen Ostblockstaaten Litauen und Weißrussland auf. Wir analysierten daher den Einfluss der folgenden Variablen auf die nationalen Suizidraten:

- Durchschnittsalter, da bekannt ist, dass in den meisten westlichen Ländern die Suizidrate in den obersten Alterskohorten am höchsten ist;

- Gini-Index, der die sozio-ökonomische Ungleichheit innerhalb einer Gesellschaft beschreibt;

- Human Development Index: dieser setzt sich aus drei Maßzahlen zusammen (Lebenserwartung, Alphabetisierungsrate im Erwachsenalter, Bruttojahres-Pro Kopf-Einkommen), die ebenfalls in die Analyse eingingen; außerdem die prozentuellen Anteile der religiösen Konfessionen in den einzelnen Staaten (s. Tab. 10).

Tab. 10 Soziodemographische und religiöse Einflussfaktoren auf die nationalen Suizidraten

\begin{tabular}{|lclc}
\hline soziodemographische Faktoren & Korrelation & religiöse Konfessionen & Korrelation \\
\hline Human Development Index &, $377^{* *}$ & Katholiken &,- 091 \\
\hline Alphabetisierungsrate &, $464^{* *}$ & Protestanten &, 047 \\
\hline Lebenserwartung &, 157 & Christlich-Orthodoxe &, 172 \\
\hline Bruttosozialprodukt pro Kopf &, $269^{* *}$ & Juden &,- 051 \\
\hline Gini-Index &,$- 470^{* *}$ & Moslems &,$- 319^{* *}$ \\
\hline Durchschnittsalter & & Hindus &, 057 \\
\cline { 2 - 4 } & & Buddhisten &, 169 \\
\cline { 2 - 4 } & \multirow{2}{*}{, $602^{* *}$} & Daoisten &, 022 \\
\cline { 2 - 4 } & & Shintoisten, Indigene &, 067 \\
\hline
\end{tabular}




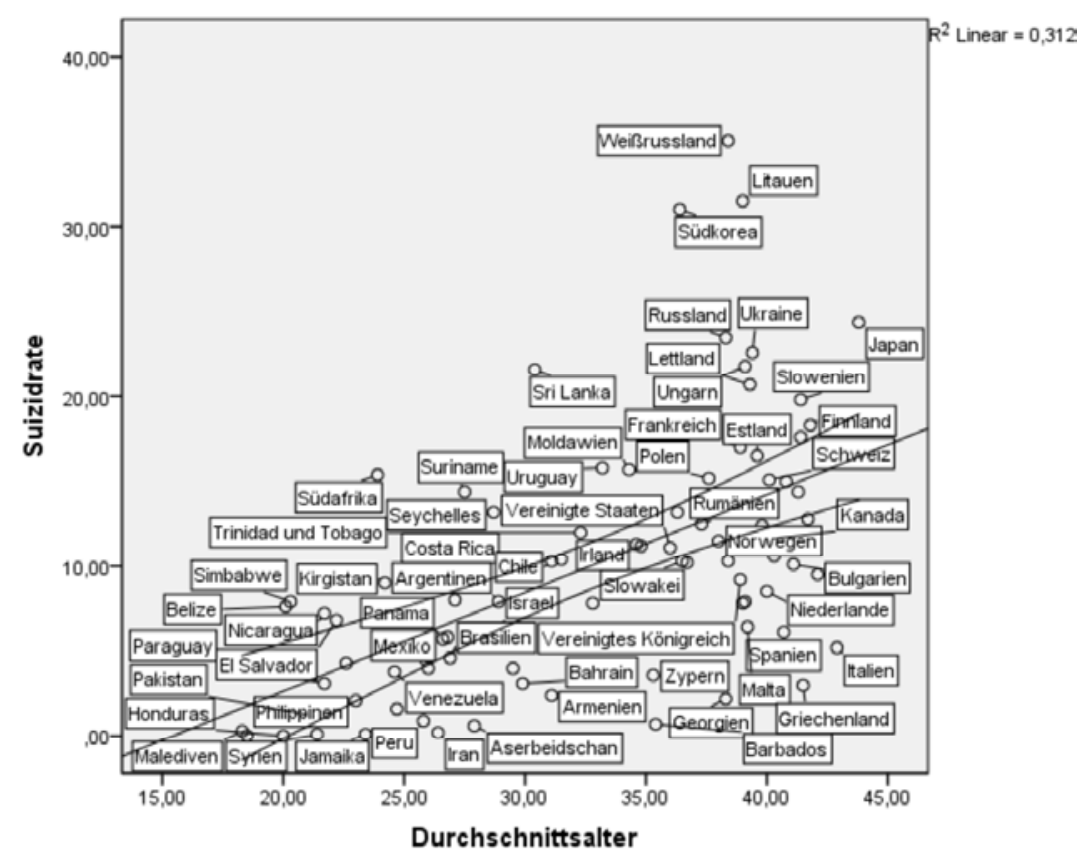

Abb. 23 Nationale Suizidraten und Durchschnittsalter

Dabei wurden die Korrelationen zwischen den nationalen Suizidraten und den soziodemographischen Daten bzw. den Angaben zur konfessionellen Verteilung getrennt gerechnet und ausgewertet. Es finden sich hochsignifikante statistische Korrelationen zwischen den Suizidraten und, ausgenommen das Durchschnittsalter, allen soziodemographischen Variablen. Besonders deutlich sind die Zusammenhänge zwischen dem Durchschnittsalter, der Alphabetisierungsrate und dem Gini-Index. Diese soziodemographischen Variablen sind allerdings nicht unabhängig voneinander. Die lineare Regressionsanalyse ergab, dass das Durchschnittsalter und das Jahresbruttosozialprodukt pro Kopf (GPI) die zentralen soziodemographischen Einflussfaktoren sind (s. Abb. 23 u. 24).

Wie aus der Literatur bekannt (De Leo 2001) ist die Suizidrate, gemessen an Anteil dieser Alterskohorte in der Gesamtbevölkerung, bei den Alten und Ältesten am höchsten. Es erscheint daher durchaus plausibel, dass Staaten mit einem höheren Durchschnittsalter gleichzeitig höhere Suizidraten aufweisen (s. Abb. 23).

Den Zusammenhang zwischen dem Jahresbruttosozialprodukt pro Kopf und den Suizidraten wiederum bestätigt die ältere Untersuchung von Conklin und Simpson (1987). Die niedrigen Suizidraten in armen Ländern können als Hinweis gelten, dass Selbsttötung bis zu einem gewissen Grad ein Wohlstandsphänomen ist. 


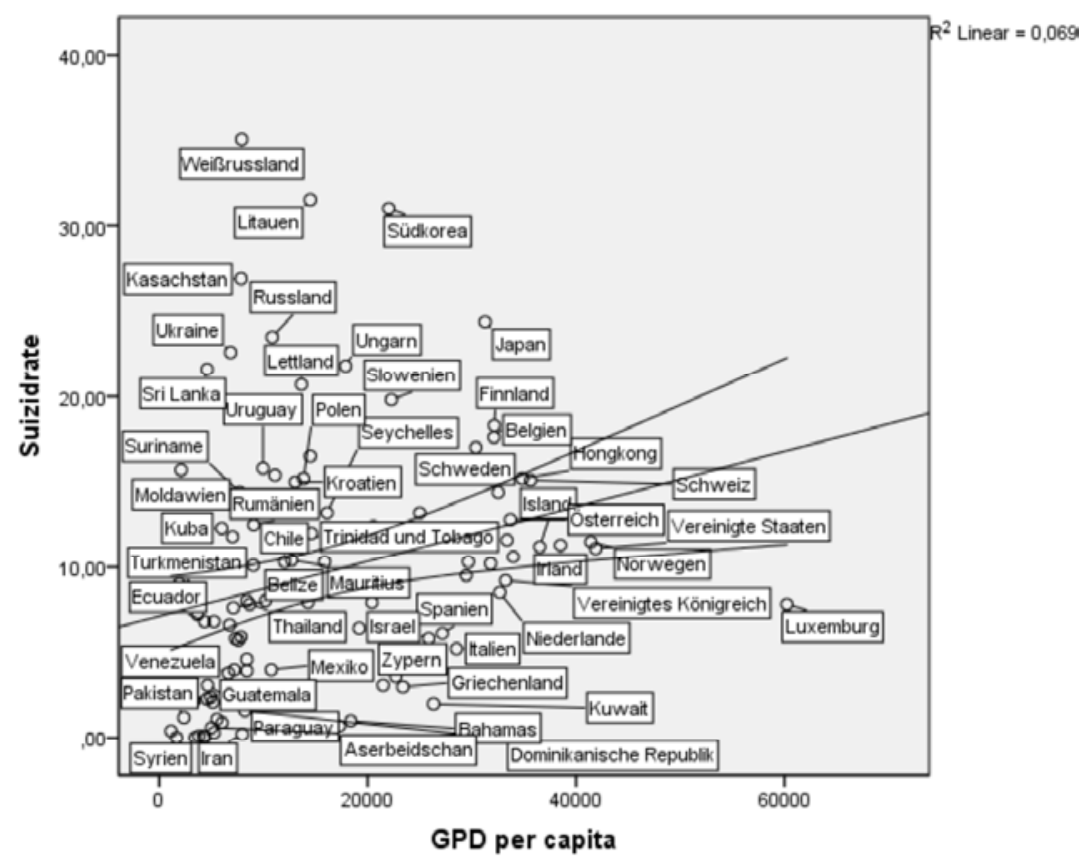

Abb. 24 Bruttonationalprodukt pro Kopf und nationale Suizidraten

\subsection{Kulturspezifische suizidale Verhaltensweisen}

Um die Hintergründe von suizidalen Verhaltensweisen in traditionellen Gesellschaften zu erforschen, analysierten Smith und Hackathorn (1982) Datensätze aus dem Human Relations Area File, einer umfassenden kulturanthropologischen Datenbank (siehe auch Lester 2008). Die Autoren fanden drei Faktoren, die $70 \%$ der Varianz der Unterschiede von Suizidraten in verschiedenen ethischen Gruppen erklären konnten:

1. Suizidraten sind in bäuerlichen Gesellschaften höher als bei Jägern und Sammlern.

2. Suizidraten sind erhöht in Gesellschaften mit expressivem oder zurückgenommenem Gefühlsausdruck.

3. Traditionelle Gesellschaften, in denen Scham und Ehre ein hoher Stellenwert eingeräumt wird, haben höhere Suizidraten als Gemeinschaften, in denen dies keine so bedeutende Rolle spielt (siehe auch Lester 1997, 1998; Kalafat u. Lester 2000).

Wie Hollan (1990) für die indonesischen Torajas, beschreibt sind auch soziale Kränkungen und Gesichtsverlust häufig auslösende Ursache für suizidale Verhaltensweisen. Ähnliches fand Leenhardt (1984) in Neukaledonien, einer melanesischen Inselgruppe. Vor allem Frauen, aber auch Männer brachten sich um, nachdem sie vom Partner oder von anderen Mitgliedern der Dorfgemein- 
schaft schlecht behandelt worden waren. Sie erwarteten, durch ihren Tod in Ahnengeister verwandelt zu werden um sich so leichter rächen zu können.

Auch in entwickelten Nationalökonomien kann suizidales Verhalten unterschiedliche Bedeutungen haben. Die auslösenden gesellschaftlichen und innerpsychischen Motive variieren stark. In Japan etwa, einem Land mit einer traditionell hohen Suizidrate, wird Selbsttötung unter gewissen Umständen als moralische Pflicht angesehen. Seppuku oder Harakiri wurde vor allem in der Kriegerklasse aber auch in der Beamtenschaft, als ehrbare Form, aus dem Leben zu scheiden, betrachtet. Historisch kann diese Praxis bis in das 12. Jahrhundert zurückverfolgt werden (Kawada 2005). Man kann vier Motive für Seppuku unterscheiden:

1. Rettung der eigenen Ehre,

2. um Vergebung bitten,

3. dem Herren in den Tod folgen und

4. als Bestrafung auferlegt.

Shingu wurde vorwiegend von jungen Liebenden praktiziert, deren Beziehung gesellschaftlich nicht erlaubt war. Diese Art, sich gemeinsam zu töten, wurde als Treuebeweis erachtet (Kannedo et al. 2005).

Eine andere kulturelle Form der Selbsttötung, die im Westen zeitweise große Beachtung fand, ist Sati (Suttee), die indische Witwenverbrennung. Dieser Brauch ist eigentlich eine Totenfolge, eine Sitte, die in alten Hochkulturen weit verbreitet war (Fisch 2005). Sie findet sich vor allem in Gesellschaften, die einen Jenseitsglauben pflegen, der davon ausgeht, dass das Jenseits ein Spiegelbild des Diesseits ist. Die Toten würden dieselbe Stellung einnehmen (können) wie im Leben. Demgegenüber findet sich der Brauch nicht in Kulturen, in denen der Platz im Jenseits aufgrund von Verdiensten im Diesseits zugewiesen wird. Die geschlechtsspezifischen Machtverhältnisse äußerten sich darin, dass die Frau ihrem Mann in den Tod nachfolgen soll, nicht allerdings umgekehrt. Dieser Brauch war allerdings nie besonders häufig und wurde von den englischen Kolonialherren schließlich verboten.

In manchen Ethnien, wie etwa den Mayas, war die Selbsttötung Teil der Kosmogonie und damit ursprünglich durchaus positiv besetzt. Ixtab, die Göttin des Suizids, wurde auf Stelen und Kodices mit einem Seil um den Hals abgebildet. Die Mayas glaubten, dass Selbstmörder, im Kampf getötete Krieger und Frauen, die im Kindbett versterben, in das Paradies aufgenommen werden. Mit der Christianisierung nahm die ursprünglich wohlmeinende Gottheit dämonische Züge an. Trotzdem ist Yukatan noch immer die mexikanische Provinz mit der höchsten Suizidrate (Bequedano 2009).

\subsection{Religion und Suizid}

Auch wenn die individuelle Religiosität in vielen Ländern nicht mehr sehr bestimmend ist, üben religiös-fundierte Wertehaltungen häufig noch immer 
einen großen gesellschaftlichen Einfluss aus. Bereits Durkheim (1997) wies auf den präventiven Einfluss von Religionen auf die Suizidalität ist hin.

In den großen monotheistischen Religionen wird Suizid abgelehnt, wobei das Christentum eine gewisse Sonderstellung einnimmt, da es kein explizites Suizidverbot in der Bibel gibt (Retterstøl u. Ekeberg 2009). Das Alte Testament schildert fünf Fälle von Suizid, ohne dabei eine wertende Stellungnahme zur Selbsttötung vorzunehmen. Erst Augustinus hat durch die Interpretation des fünften Gebotes „Du sollst nicht töten“ auch einen Bezug zur Selbsttötung hergestellt und Suizid somit als Handlung wider Gottes Willen definiert.

Im Judentum ist das Tötungsverbot explizit in der Thora festgehalten (Orbach u. Rabinowitz 2009). Das Leben wird als Leihgabe von Gott gesehen, der Mensch besitzt kein absolutes Anrecht auf seinen Körper. Der Suizid wurde lange Zeit sogar dem Mord gleichgesetzt.

Im Islam findet man im Koran selbst keine explizite Aussage zum Suizid, allerdings wird ein Verbot, Menschen zu töten, in verschiedenen Suren zum Ausdruck gebracht, die auch auf den Suizid bezogen werden können (Schultz 2009). In den Hadithen hingegen findet sich eine ganze Reihe von Traditionen, die sich dieses Themas annehmen:

„Ein Mann war verletzt und tötete sich selbst. Und Gott sagte: mein Knecht ist mir zuvor gekommen, ich verbot ihm das Paradies“ (al-Buhari: Kitãb al-ğanã’iz, Kapitel 84)

„Wer sich selbst mit einem Stück Eisen tötet wird von ihm im Feuer der Hölle gepeinigt“ (Ibn Hanbal)

„Wer sich von einem Berg stürzt und sich selber tötete, wird im Feuer der Hölle sein und in ihm auf alle Ewigkeit stürzen und ewig in ihm bleiben. Und wer Gift trinkt und sich selbst tötet, wird sein Gift in seiner Hand im Feuer der Hölle auf alle Ewigkeiten trinken und in ihm ewig bleiben. Und wer sich mit dem Eisen selbst tötet, wird im Höllenfeuer auf alle Ewigkeiten sein Eisen durch seine Hand in dem Bauch geführt bekommen und in ihm ewig bleiben“ (al-Buhari: Kitâb al-tibb, Kapitel 59)

Übereinstimmend zeigen diese Passagen eine ablehnende Haltung gegenüber dem Suizid, und es kristallisiert sich deutlich die Aussage heraus, dass es verboten ist, sich selbst das Leben zu nehmen (Okasha u. Okasha 2009).

Der Hinduismus nimmt in den vedischen Schriften (um 1.200 v. Chr.) noch keine eindeutige Position zum Suizid ein (Vijakumar 2009). Bereits in den Upanischaden (700-500 v. Chr.) hingegen wird Suizid kategorisch abgelehnt:

„Er, der sich sein Selbst nimmt, kommt nach dem Tod in eine sonnenlose Gegend, die in undurchdringliche Finsternis gehüllt ist“"(Isavasya Upanishad)

Auch im Dharmashastra (das Hindu Buch der Regeln und der Ethik, 9oo700 v. Chr.) wird der Suizid verdammt. Allerdings gibt es besondere Formen des erlaubten ritualisierten Suizids, wie Sati (Suttee), die bereits erwähnte 
indische Witwenverbrennung, die im modernen Indien nicht mehr praktiziert wird, sowie Mahaparasthana, eine religiöser Suizidform, bei der vor allem alte Menschen, die sich dem Tod nahe wissen, eine Wanderung nach der heiligen Stadt Kailash unternehmen, weitgehend ohne Nahrung zu sich zu nehmen. Wenn der Reisende durch Entkräftigung stirbt, ist dies der Ausstieg aus dem Kreislauf der Wiedergeburten.

Im Buddhismus wird Suizid zwar nicht direkt verboten, aber als unsinnige Handlung angesehen, da sie das Karma verschlechtert und man somit im nächsten Leben nochmals den unveränderten Problemen ausgesetzt sein wird (Prompta u. Thomyangkoon 2009). Suizid stellt auf jeden Fall, egal aus welchen Beweggründen, immer eine unheilvolle Tat für das Karma dar.

Zhuangzi (Tschung Tse, etwa 369-285 v. Chr.), ein zentraler Autor des Daoismus, nimmt in seinem Werk „Das wahre Buch vom südlichen Blütenland " eine indifferente Haltung ein. Obwohl er davon ausgeht, dass das Leben nicht immer besser ist als der Tod, befürwortet er den Suizid nicht, da er ein Zeichen für eine Überbewertung einer einzelnen Idee sei.

Auch wir gingen dem potenziellen Einfluss von Religionen auf die nationalen Suizidraten nach. Im Speziellen untersuchten wir die Auswirkung der prozentuellen Verteilung der religiösen Konfessionen (s. Tab. 10). Die Daten dazu wurden dem jährlich erscheinenden Fischer Weltalmanach entnommen (Redaktion Weltalmanach 2009). Zwischen den Suizidraten und dem prozentuellen muslimischen Bevölkerungsanteil zeigte sich eine negative Korrelation, während Staaten mit einem hohen Prozentsatz an Personen ohne religiöses Bekenntnis hohe Selbsttötungsraten aufwiesen. Bei verschiedenen christlichen Konfessionen, im Judentum, im Hinduismus, im Buddhismus und im Daoismus waren diese Korrelationen nicht nachweisbar.

Staaten mit muslimischen Minderheiten oder ohne Bevölkerungsteile, die sich zum Islam bekennen, zeigen eine breite Streuung, Länder mit muslimischer Mehrheitsbevölkerung mit der Ausnahme von Kasachstan niedrige Suizidraten (s. Abb. 25).

Damit können die Ergebnisse älterer Untersuchungen bestätigt werden, die ebenfalls niedrige Suizidraten in Islamischen Ländern fanden (zur Übersicht: Lester 2006).

Die Verbindung zwischen hohen Suizidraten und hohem Prozentanteil von Menschen ohne religiöses Bekenntnis findet sich vor allem in den Staaten des ehemaligen Ostblocks (Ungarn, Tschechien) und den Nachfolgestaaten der UDSSR (Litauen, Weißrussland, Russland) (s. Abb. 26). Gleichzeitig sind dies mehrheitlich Länder, die nach dem Zerfall des Kommunismus eine Umstellung von einer Planwirtschaft zu einer neoliberalen Marktwirtschaft im Zeitraffertempo durchlaufen haben. Die Folge war eine Polarisierung in eine neue Oberschicht Reichgewordener einerseits und in zahlreiche Modernisierungs- 


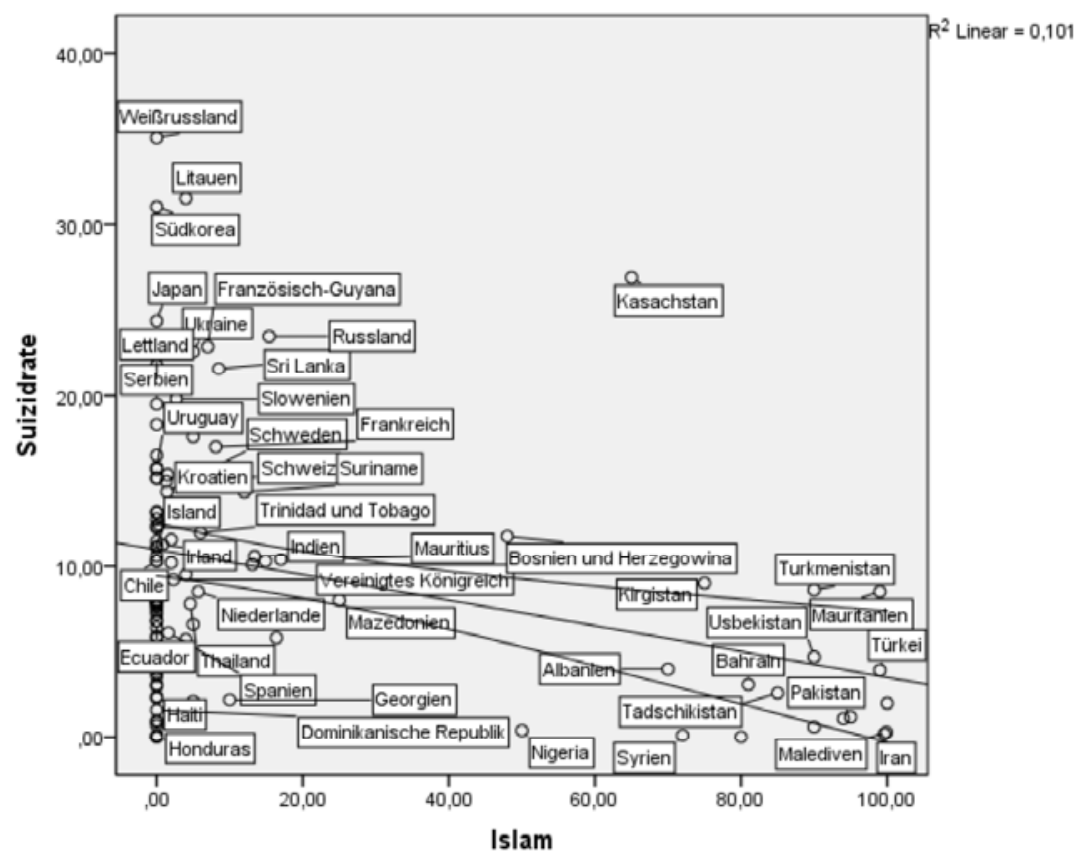

Abb. 25 Nationale Suizidraten und prozentueller Anteil von Einwohnern mit muslimischem Bekenntnis

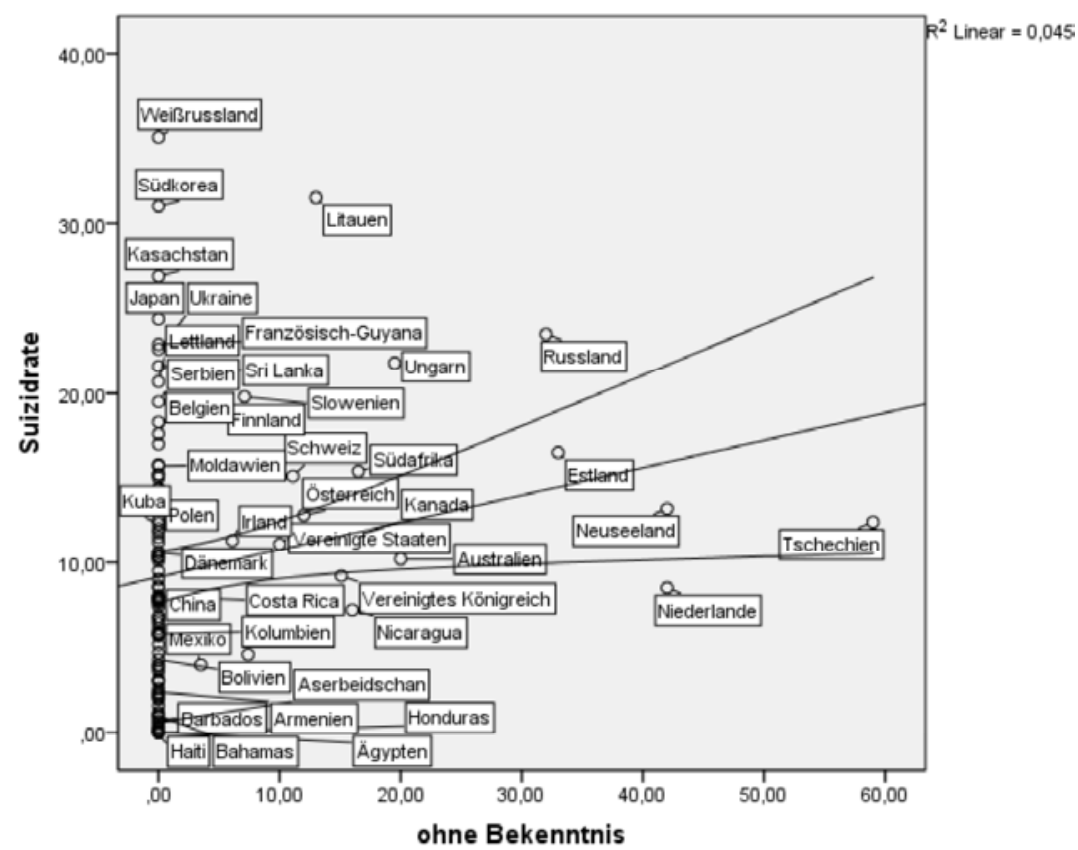

Abb. 26 Nationale Suizidraten und prozentueller Anteil von Einwohnern ohne Konfession 
verlierer andererseits. Schattenseiten der neuen Freiheiten waren häufig eine Entsolidarisierung der Gesellschaft und der Verlust tragender Werte. Bereits Durkheim (1997) postulierte, dass Suizidraten in Zeiten mit anomischem Wertezerfall gegenüber stabilen Perioden ansteigen.

Dies lässt sich auch daran erkennen, dass ehemalige kommunistische Länder mit einer starken christlichen Tradition wie Polen, die Slowakei oder Rumänien deutlich niedrigere Suizidraten aufweisen als die oben genannten Staaten. Weiter kompliziert wird die Interpretation der hohen litauischen, weißrussischen und ungarischen Selbsttötungsraten durch die starken finnischugrischen Bevölkerungsanteile in diesen Ländern, für die ja eine genetische Belastung postuliert wird.

\subsection{Soziokultureller Umgang mit Suizid}

Die von der WHO veröffentlichten Zahlen sind allerdings mit einer gewissen Skepsis zu betrachten. In einigen Ländern ist das Thema Suizid noch immer ein so großes Tabu, dass keine offiziellen Zahlen existieren. Das ist vor allem in vielen afrikanischen Ländern, aber auch in Indonesien, in einigen asiatischen Staaten und in Teilen Lateinamerikas der Fall. Einer der Hauptgründe dafür ist die starke soziale Stigmatisierung der Familien von Suizidanten. In einigen afrikanischen Ländern, so zum Beispiel in Nigeria, verschlechtern sich die Heiratschancen der weiblichen Familienangehörigen von Suizidanten drastisch. In anderen Ländern wie etwa Pakistan ist ein Suizidversuch eine mit Gefängnisstrafe bedrohte Handlung. Suizid wird als eine gegen Gott gerichtete Handlung gesehen, da nur Gott allein über Leben und Tod seiner Schöpfungen zu entscheiden hat. Manchmal wird eine Selbsttötung mit Geisteskrankheit gleichgesetzt, was ebenfalls zu einer sozialen Stigmatisierung der Hinterbliebenen führt. Oft wird daher versucht, einen Suizid als Unfall oder als überraschenden Todesfall nach akuter Krankheit darzustellen.

Aus schriftlosen Gesellschaften, aber auch aus schriftlichen Überlieferungen von der Antike bis in die frühe Neuzeit sind Ängste vor den Seelen der Menschen, die durch die eigene Hand verstorben sind, bekannt. So verbrennen die Baganda aus Zentralafrika den Körper eines Erhängten mit dem Holz des Baums, den er dafür benützt hat, an einer Wegkreuzung. Es ist die Aufgabe der Frauen des Stammes, die Asche des Toten mit Erde zu bedecken, da Erzählungen besagen, dass dieser sonst in die Körper der Frauen eindringen und wieder aufleben kann.

Von den Ewe aus Togo wird berichtet, dass sie den Erhängten samt dem Ast, an dem er aufgehängt war, solange über Dornen schleifen, bis der Körper zerfetzt ist. Erst dann begraben sie den Leichnam an einer besonders abgelegenen Stelle. Eine andere Beschreibung schildert, wie die Ewe dem Leichnam einen Pfahl durch die Brust stoßen und ihn anschließend verscharren. Durch diese Bräuche soll die Rückkehr des rachsüchtigen Toten zu den Lebenden verhin- 
dert werden (Minois 1996). Auch in anderen afrikanischen Gesellschaften wird die Leiche oftmals rituell verstümmelt, letztlich aber immer mit dem Ziel, eine Wiederauferstehung und Verfolgung der Lebenden zu verhindern. Vom Brechen der Schenkelknochen, Abreißen der Ohren bis zum Abschlagen der Hände, jeder dieser Verstümmelungen liegt die Vorstellung zugrunde, dass der so geschändete Leichnam keinen Schaden mehr anrichten kann. Dies ist nur ein kleiner Ausschnitt aus dem breiten Spektrum ethnographischer Schilderungen. Weltweit sind aus nahezu allen Ethnien spezielle Bestattungsrituale für die Leichname von Suizidanten bekannt, da diese Todesart als eine besonders unheilvoll angesehen wird (Prinz 1998).

\section{Zusammenfassung}

Dem Leben durch eigene Hand ein Ende zu setzen ist eine der Handlungsfreiheiten, durch die sich der Mensch vom Tier unterscheidet. Voraussetzung dafür ist das Wissen über die eigene Endlichkeit und die Unvorhersehbarkeit des Todeszeitpunkts. Suizidale Handlungen finden sich dementsprechend in allen uns bekannten Gesellschaften. Der vollendete Suizid ist zumeist das Endresultat von komplex interagierenden biologischen, psychologischen, psychopathologischen und sozialen Faktoren. Obwohl Selbsttötung somit als anthropologische Konstante zu betrachten ist, unterscheiden sich die weltweit vorgefundenen Suizidraten regional erheblich. Sowohl biologische, demographische und soziokulturelle Faktoren als auch religiöse (oder atheistische) Wertehaltungen beeinflussen in Krisensituationen die individuelle Entscheidung für oder gegen das eigene Leben.

\section{Literatur}

Arango V, Huang YY, Underwood MD, Mann || (2003) Genetics of the serotonergic system in suicidal behavior. | Psychiatr Res 37, 375-386

Arsenault-Lapierre G, Kim C, Turecki G (2004) Psychiatric diagnoses in 3275 suicides: a meta-analysis. BMC Psychiatry 4, 37

Audenaert K, Peremans K, Goethals I, van Heeringen C (2006) Functional imaging, serotonin and the suicidal brain. Acta Neurol Belg 106, 125-131

Baquedano G (2009) Maya religion and traditions: influencing suicide prevention in contemporary Mexico. In: Wasserman D, Wasserman C (Hrsg.) Oxford textbook of suicidology and suicide prevention. 77-84. 0xford University Press New York

Bondy B, Buettner A, Zill P (2006) Genetics of suicide. Mol Psychiatry 11, 336-351

Brent DA, Melhem N (2008) Familial transmission of suicidal behavior. Psychiatr Clin North Am 31, 157-177

Brunner I, Bronisch I (1999) Neurobiologische Korrelate suizidalen Verhaltens. Fortschr Neurol Psychiatr 67, 391-412

Canetto SS (2008) Women and suicidal behavior: a cultural analysis. Am I Orthopsychiatry 78, 259-266

Cheng ATA (1995) Mental illness and suicide. Arch Gen Psychiatry 52, 594-603

Cheng ATA (1997) Personality disorder and suicide. British Journal of Psychiatry 170, 441-446

Chiao JY, Blizinsky KD (2010) Culture-gene coevolution of individualism-collectivism and the serotonin transporter gene. Proc Biol Sci 277:529-537

Conklin GH, Simpson ME (1987) The family, socioeconomic development and suicide. Journal of Comparative Family Studies 18, 99-111

Conrad RD, Kahn MW (1974) An epidemiological study of suicide and attempted suicide among the Papago indians. Am J Psychiatry 131, 69-72 
Courtet P, Jollant F, Castelnau D, Buresi C, Malafosse A (2005) Suicidal behavior: relationship between phenotype and serotonergic genotype. Am I Med Genet C Semin Med Genet 15, 25-33

De Leo D (Hrsg.) (2001) Suicide and euthanasia in older adults. A transcultural journey. Hogrefe u. Huber Publishers Seattle Toronto Bern Göttingen

Durkheim E (1997) Der Selbstmord. Suhrkamp Frankfurt am Main

Feldmann K (2001) Suizid: sozialwissenschaftliche Theorien, wissenssoziologische und ideologiekritische Überlegungen. Skript Univ. Hannover

Fisch I (2005) Die indische Witwenverbrennung: Tötung oder Selbsttötung? In: Bähr A, Medick H (Hrsg.) Sterben von eigener Hand. Selbsttötung als kulturelle Praxis. 309-332. Böhlau Köln Wiemar Wien

Freud S (1999a) Gesammelte Werke. Totem und Tabu. 9. Band. Fischer Taschenbuch Verlag Frankfurt am Main

Freytag R, Giernalezyk T (2001) Geschlecht und Suizidalität. Vandenhoeck u. Ruprechtverlag Göttingen

Gibbs IP, Martin W (1964) Status integration and suicide. University of Oregon Press Eugene

Halbwachs M (1978/1930) The causes of suicide. Routledge and Kegan London

Hawgood I, De Leo D (2008) Anxiety disorders and suicidal behaviour: an update. Curr Opin Psychiatry 21, 51-64

Henseler H (2000) Narzisstische Krisen. Zur Psychodynamik des Selbstmordes. Westdeutscher Verlag Wiesbaden.

Hollan D (1990) Indignant suicide in the Pacific: an example from the Toraja Highlands of Indonesia. Cult Med Psychiatry 14, 365-379

Hor K, Taylor M (2010) Suicide and schizophrenia: a systematic review of rates and risk factors. I Psychopharmacol 24(4 Suppl), 81-90

Ji I, Kleinman A, Becker AE (2001) Suicide in contemporary China: a review of China's distinctive suicide demographics in their sociocultural context. Harv Rev Psychiatry 9, 1-12

Kalafat J, Lester D (2000) Shame and suicide: a case study. Death Stud 24,157-162

KannedoY, Yamasaki A, Arai K (2009) The Shinto religion and suicide in Japan. In: Wasserman D, Wasserman C (Hrsg.) Oxford textbook of suicidology and suicide prevention. 37-41. Oxford University Press New York

Kawada I (2005) Moral and aesthetic aspects of suicide among the Japanese: Seppuku, self-immolation and Shinju, double suicide of lovers. In: Bähr A, Medick H (Hrsg.) Sterben von eigener Hand. Selbsttötung als kulturelle Praxis. 277-290. Böhlau Köln Wiemar Wien

Krysinska K, Lester D (2010) Post-traumatic stress disorder and suicide risk: a systematic review. Arch Suicide Res 14, 1-23

Leenhardt M (1984) Do Kamo. Die Person und der Mythos in der melanesischen Welt. Ullstein Frankfurt am Main

Lester D (1992) The dexamethasone suppression test as an indicator of suicide: a meta-analysis. Pharmacopsychiatry $25,265-270$

Lester D (1997) The role of shame in suicide. Suicide Life Threat Behav 2, 352-361

Lester D (2004) Blood types and national suicide rates. Crisis 25,140

Lester D (2006) Suicide and islam. Arch Suicide Res 10, 77-97

Lester D (2008a) Suicide and culture. WCPRR 3, 51-68

Lester D (2008b) Suicide among indigenous peoples: the usefulness of the Human Relations Area Files. Crisis 29, $49-51$

López JF, Palkovits M, Arató M, Mansour A, Akil H, Watson SI (1992) Localization and quantification of proopiomelanocortin mRNA and glucocorticoid receptor mRNA in pituitaries of suicide victims. Neuroendocrinology 56, 491-501

Mann II (1998) The neurobiology of suicide. Nature Medicine 4, 25-30

Mann II, Arango VA, Avenevoli S, Brent DA, Champagne FA, Clayton P, Currier D, Dougherty DM, Haghighi F, Hodge SE, Kleinman I, Lehner T, McMahon F, Mościcki EK, Oquendo MA, Pandey GN, Pearson I, Stanley B, Terwilliger I, Wenzel A (2009) Candidate endophenotypes for genetic studies of suicidal behavior. Biol Psychiatry 1, 56-63

Menninger K (1989) Selbstzerstörung. Psychoanalyse des Selbstmords. Suhrkamp Frankfurt am Main

Minois G (1996) Geschichte des Selbstmords. Artemis u. Winkler Verlag Düsseldorf Zürich 
Mullany B, Barlow A, Goklish N, Larzelere-Hinton F, Cwik M, Craig M, Walkup IT (2009) Toward understanding suicide among youths: results from the White Mountain Apache tribally mandated suicide surveillance system, 2001-2006. Am I Public Health 99, 1840-1848

Ogden M, Spector MI, Hill CA (1970) Suicides and homicides among Indians. Public Health Rep 85, 75-80

Okasha A, Okasha T (2009) Suicide and Islam. In: Wasserman D, Wasserman C (Hrsg.) Oxford textbook of suicidology and suicide prevention. 49-55. Oxford University Press New York

Orbach I, Rabinowitz A (2009) Suicide in Jewish scriptures. In: Wasserman D, Wasserman C (Hrsg.) Oxford textbook of suicidology and suicide prevention. 43-47. Oxford University Press New York

Pedersen NL, Fiske A (2010) Genetic influences on suicide and nonfatal suicidal behavior: twin study findings. Eur Psychiatry 25, 264-267

Pfeiffer W (1994) Transkulturelle Psychiatrie. Ergebnisse und Probleme. Georg Thieme Verlag Stuttgart

Prinz A (1998) Über den Umgang mit Leichen bei fremden Völkern. In: Stefenelli N (Hrsg.) Körper ohne Leben. Begegnungen und Umgang mit Toten. Böhlau Verlag Wien Köln Weimar

Prompta S, Thomyangkoon P (2009) A Buddhist perspective on suicide. In: Wasserman D, Wasserman C (Hrsg.) Oxford textbook of suicidology and suicide prevention. 27-35. Oxford University Press New York

Redaktion Weltalmanach (2009) Der Fischer Weltalmanach 2010: Zahlen Daten Fakten. Fischer Frankfurt am Main

Retterstøl N, Ekeberg 0 (2009) Christianity and suicide. In: Wasserman D, Wasserman C (Hrsg.) Oxford textbook of suicidology and suicide prevention. 57-62. Oxford University Press New York

Ringel E (1998) Selbstmord. Appell an die anderen. Kaiser Taschenbücher Verlag Güthersloh

Rujescu D, Giegling I (2010) The genetics of neurosystems in mental ill-health and suicidality: beyond serotonin. Eur Psychiatry. 25, 272-274

Schultz D (2009) Dürfen Muslime sich selbst töten? Das Suizid-Verbot in der islamischen Theologie und dem islamischen Recht. Tectum Verlag Marburg

Smith DH, Hackathorn L (1982) Some social and psychological factors related to suicide in primitive societies: a cross-cultural comparative study. Suicide Life Threat Behav 12, 195-211

Souery D, Oswald P, Linkowski P, Mendlewicz I (2003) Molecular genetics in the analysis of suicide. Ann Med 35, 191-196

Stack S (2000a) Suicide: A 15-year review of the sociological literature. Part I: Cultural and economic factors. Suicide Life Threat Behav 30, 145-162

Stack S (2000b) Suicide: A 15-year review of the sociological literature. Part II: modernization and social integration perspectives. Suicide Life Threat Behav 30, 163-176

Tsai SI, Hong CI, Liou Y] (2010) Recent molecular genetic studies and methodological issues in suicide research. Prog Neuropsychopharmacol Biol Psychiatry 23

United Nations Development Program (2011) Human Development Report 2010. Hoechstetter New York

van Heeringen K (2003) The neurobiology of suicide and suicidality. Can I Psychiatry 48, 292-300

Vijayakumar L (2009) Hindu religion and suicide in India. In: Wasserman D, Wasserman C (Hrsg.) Oxford textbook of suicidology and suicide. 19-25. Oxford University Press New York

Voracek M, Loibl LM (2007) Genetics of suicide: a systematic review of twin studies. Wien Klin Wochenschr 119, $463-475$

Voracek M, Loibl LM (2008) Consistency of immigrant and country-of-birth suicide rates: a meta-analysis. Acta Psychiatr Scand 118, 259-271

Voracek M, Loibl LM, Kandrychyn S (2007) Testing the Finno-Ugrian suicide hypothesis: replication and refinement with regional suicide data from eastern Europe. Percept Mot Skills 104, 985-994

Yip PS, Liu KY, Hu J, Song XM (2005) Suicide rates in China during a decade of rapid social changes. Soc Psychiatry Psychiatr Epidemiol 40, 792-798 